\title{
Facet joint degeneration adversely affects ultrasound imaging of the lumbar spine
}

\author{
Karthikeyan Kallidaikurichi Srinivasan', Hong Kuan Kok², Mona Mubarak ${ }^{3}$, William \\ Torregianni $^{2}$, Rob Whitty ${ }^{1}$
}

${ }^{1}$ Department of Anaesthesia and Intensive Care, Tallaght Hospital, Dublin, Ireland, ${ }^{2}$ Department of Radiology, Tallaght Hospital, Dublin, Ireland, ${ }^{3}$ Department of Anaesthesia and Pain Medicine, Royal Orthopaedic Hospital, Birmingham, UK

\begin{abstract}
Aim: Ultrasound of neuraxis can be used to identify the best possible inter-spinous space to perform neuraxial block. The aim of this study was to assess the anatomical correlation between neuraxial ultrasound and magnetic resonance imaging (MRI). Material and method: Twenty-one patients who underwent MRI of the lumbar spine had ultrasound of lumbar neuraxis performed by an experienced operator. Each lumbar interspinous space was graded on ultrasound as good [posterior complex (ligamentum flavum and duramater) and anterior complex (posterior longitudinal ligament) visible], intermediate (either anterior complex or posterior complex visible) or poor (both anterior complex and posterior complex not visible) in both the transverse median (TM) and paramedian sagittal oblique (PSO) plane. Pre-determined MRI parameters were measured by a radiologist blinded to sonographic findings at each inter-spinal level. Results: Seventy-eight lumbar interspinous spaces were evaluated. There was a significant association $(\mathrm{p}<0.004)$ between the facet joint degeneration on MRI and the poor ultrasound view in the transverse median (TM) group. The odds of obtaining a poor view in TM plane was 7 times higher (95\% CI $1.7-28.9, \mathrm{p}=0.007$ ) in the presence of facet joint degeneration. None of the other variables had a significant association with a poor neuraxial view in the TM plane. Poor views in the parasagittal oblique (PSO) plane did not correlate with any of the variables measured on MRI. Conclusion: Facet joint degeneration is a major contributing factor to poor neuraxial ultrasound views in the TM plane.
\end{abstract}

Keywords: neuraxial ultrasound; MRI; facet joints

\section{Introduction}

Good visibility of ligamentum flavum-dura mater complex (Posterior Complex - PC) and posterior longitudinal ligament (Anterior Complex - AC) in neuraxial ultrasound is a predictor of successful neuraxial block [1]. Unfortunately, a high negative predictive value (approximately $30 \%$ [2]) limits its clinical value in patients deemed to be at risk of a technically difficult neuraxial block.

Received 02.09.2018 Accepted 14.01.2019

Med Ultrason

2019, Vol. 21, No 2, 131-135

Corresponding author: Dr. Karthikeyan Kallidaikurichi Srinivasan, FCARCSI, FRCA, MD

Department of Anaesthesia and Intensive Care, Tallaght Hospital, Dublin

E-mail: karthik@outlook.ie
Poor visualisation of ligamentum flavum-dura mater complex may be due to one of two reasons: i) attenuation of the sonographic beam by anatomic structures such as ligament calcification, facet joint hypertrophy, narrow interspinous spaces etc. and ii) absence or anatomic alteration of the structure (eg. surgical laminectomies, absence or gaps in ligamentum flavum).

Distinguishing these possible contributors have practical implications depending on the neuraxial procedure performed. If a poor view is obtained because of sonographic attenuation, it might translate to difficulty in performing the neuraxial block. By contrast, if it is due to absence of the target structure, it may still be possible to successfully perform a spinal anaesthetic or dural tap depending on the block intended. Hence, it is relevant to look for anatomical reasons behind poor neuraxial ultrasound views.

The aim of this study is to assess the anatomical correlation between neuraxial ultrasound and magnetic reso- 
nance imaging (MRI) of the lumbosacral spine at various lumbar interspinous levels and to identify factors contributing to poor neuraxial ultrasound imaging.

\section{Material and methods}

A prospective observational study was performed in a tertiary university hospital between January 2013 to July 2013. Ethical committee approval was obtained from the local Ethics Committee (Tallaght Hospital/St. James's Hospital Joint Research Ethics Committee REC) and informed written consent was obtained from all participants. All patients over the age of 18 years who underwent MRI of the lumbosacral spine were eligible for inclusion. Exclusion criteria included patients with previous spinal surgery, gross spinal deformities and body mass index $(\mathrm{BMI})>40$. Both MRI and neuraxial ultrasound were performed sequentially on the same day.

\section{MRI protocol}

Imaging was performed with patient in supine, on a 1.5T MRI system (Symphony, Siemens AG, Erlangen, Germany) using a circularly polarised spinal array coil. T1 and T2-weighted sagittal and selected T2-weighted axial sequences through the lumbosacral spine were obtained. Images were reviewed in consensus by two experienced radiologists (HKK and WCT) on a dedicated PACS workstation (Syngo Studio Advanced V36A, Siemens AG, Erlangen, Germany). Measurements were performed on T2-weighted sagittal sequences (TR 3930.0 ms, TE $99.0 \mathrm{~ms}$, matrix $320 \times 288$, slice thickness 4.0 $\mathrm{mm}$ ) and included the oblique subcutaneous fat thickness, interspinous distance, thecal sac diameter and posterior longitudinal ligament thickness. Further measurements were performed on T2-weighted axial sequences (TR $6570.0 \mathrm{~ms}$, TE $95.0 \mathrm{~ms}$, matrix 320x165, slice thickness $1.5 \mathrm{~mm}$ ) at all available levels including epidural fat thickness, distance from the skin surface to the posterior longitudinal ligament, thecal sac diameter, ligamentum flavum thickness, midline fusion of ligamentum flavum and paraspinal muscle thickness. In addition, the presence or absence of facet joint degenerative change was graded as either present or absent. Both radiologists were blinded to ultrasound findings.

\section{Ultrasound protocol}

Ultrasound scanning of the lumbar interspinous spaces was performed on the same day with curvilinear 2-5 MHz probe (P07576, SonoSite Inc, Bothell, WA, 98021, USA). Ultrasound scanning was done by one of the three experienced operators (RW, KKS, MM) each with experience of more than 100 neuraxial ultrasounds. Scanning was performed with patients in the sitting position with both feet supported by a foot stool. Patients were requested to hug a pillow and arch their back. At each lumbar interspinous level (L1-2 to L5-S1) the best possible transverse median (TM) and paramedian sagittal oblique (PSO) view of posterior complex (PC) and anterior complex (AC) was obtained. The images were recorded for subsequent review. Two authors (KKS and $\mathrm{MM}$ ) independently graded the images based on the visibility of AC and PC. When both AC and PC were clearly visible, it was graded as good. If either AC or PC was not clearly visible, it was graded as intermediate. When both $\mathrm{AC}$ and $\mathrm{PC}$ was not visible, it was graded as poor (fig 1). Readers were blinded to MRI findings. If there was a disagreement in the image grading between the two observers, the third observer (RW) was requested to review the images. Decision on the image grading was made based on overall consensus.

\section{Statistical analyses}

For numerical data mean \pm standard deviation (SD) were calculated. Continuous data was analysed for normality using the Shapiro-Wilk test. Students t-tests were used to compare normally distributed continuous data. Three variables were normally distributed - skin to PLL distance, thecal sac diameter and para-spinal muscle thickness. All other variables were not normally distributed. Nonparametric data were compared by the MannWhitney U test and categorical data were compared using the chi-square test. Logistic regression was used to analyse the degree of correlation between the variables that differed significantly between groups. Statistical analyses were performed using SPSS (Version 20, IBM Corporation, Armonk, NY).

\section{Results}

Twenty-one patients (mean age 52.4 \pm 12.4 years, weight $78.9 \pm 1.7 \mathrm{~kg}$, height $161.6 \pm 9.6 \mathrm{~cm}$, BMI $30.1 \pm 3$ ) were included in the study and a total of 78 interspinous spaces were evaluated with ultrasound and MRI. The female to male ratio was 1:2. MRI data was not available for all interspinous levels: the L3-4, L4-5 and L5-S1 interspaces were evaluated in all 21 patients but L2-3 was evaluated in only seven interspaces and L1-2 in eight interspaces. The grading of the ultrasound aspect in TM and PSO views is shown in table I and the presence of facet joint degeneration in ultrasound TM view in table II.

Facet joint degeneration was found more frequently on MRI in TM poor view group compared to intermediate and good view groups $(\mathrm{p}=0.004)$. Failure of midline ligamentum flavum fusion was seen in only 2 of 78 levels (one at L3-4 and L4-5 respectively in one patient). There was no difference $(\mathrm{p}=0.35)$ in the ligamentum flavum fusion between the two groups (TM poor view versus good/ 


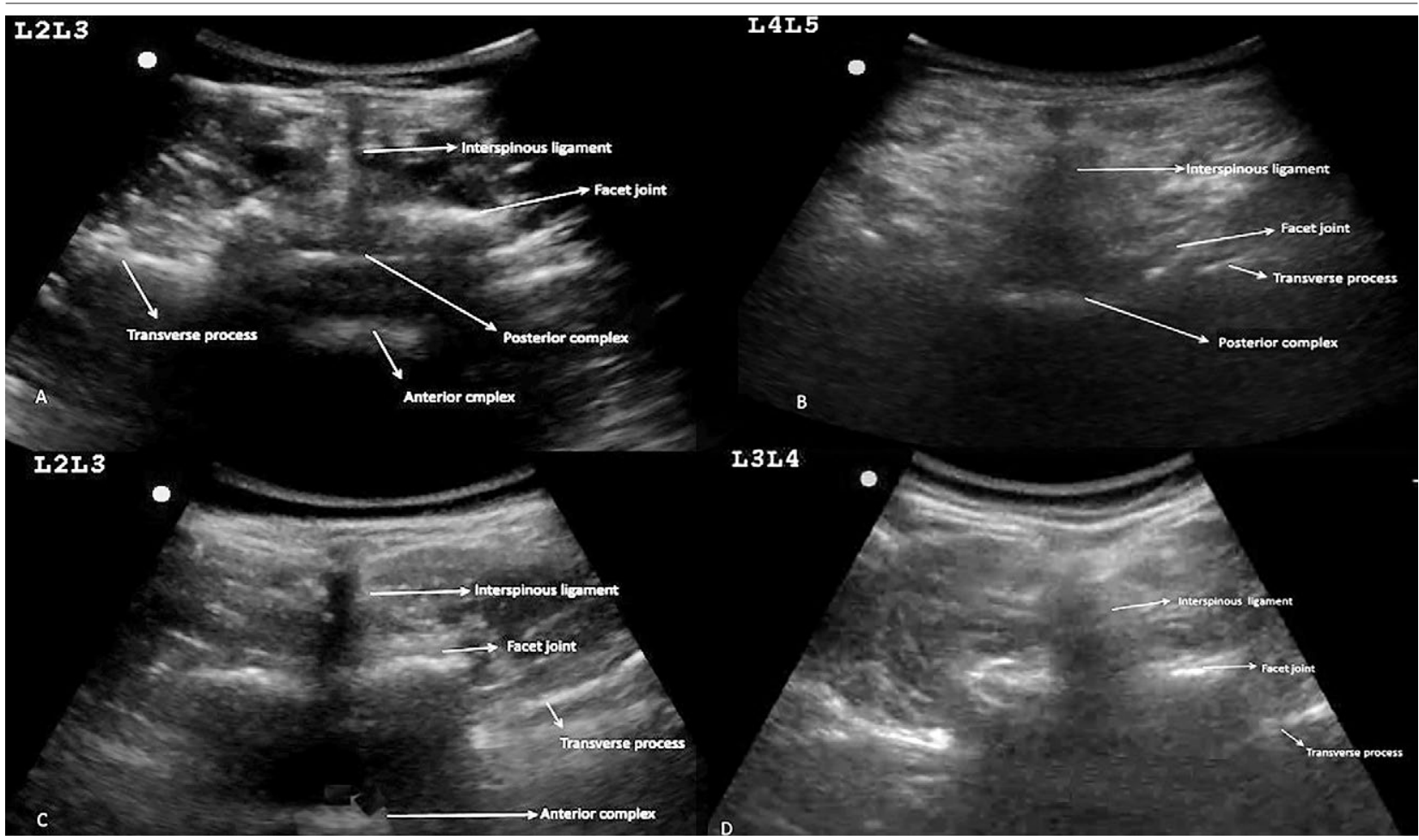

Fig 1. Grading of neuraxial ultrasound, transverse median (TM) views: A) Good view - both posterior and anterior complex are visible; B) Intermediate view - only posterior complex is visible; C) Intermediate view - only anterior complex is visible; D) Poor view - no visibility of either anterior or posterior complex

intermediate views). The adjusted logistic regression model for poor view in TM plane was positively associated with facet joint degeneration. The odds ratio of obtaining a poor view in TM plane is 7.0 (95\% CI 1.7-28.9, $\mathrm{p}=0.007)$ in the presence of facet joint degeneration on MRI. Comparison of continuous variables between poor TM views versus others (good and intermediate views in TM plane) are presented in table III. Age (median 54 vs 47 years, $\mathrm{p}=0.016$ ), weight (median $80 \mathrm{vs} 77 \mathrm{~kg}, \mathrm{p}=0.03$ ) and BMI (32.1 vs $29.1 \mathrm{~kg} / \mathrm{m}^{2}, \mathrm{p}=0.01$ ) were higher in patients with poor ultrasound views.

\section{Discussions}

Spinal ultrasonography is challenging due to the anatomic nature and layout of the spine. As the ultrasound beam passes through the bony spinal cage, it is subjected to multiple artefacts which significantly limits the ability of ultrasound beam to visualise the structures within the canal. Two such artefacts might be relevant in helping us to understand the role played by facet joint degeneration in causing a poor ultrasound view: refraction shadowing (supplemental figure 1, on the journal site) and scattering.

At the margins of a structure with different acoustic impedance compared to the tissue around it and with a
Table I. The ultrasound grading of the imagines obtained in transverse median (TM) and paramedian sagittal oblique (PSO) views

\begin{tabular}{llll}
\hline Parameters & Good view & Intermediate view & Poor View \\
\hline TM view & $24(30.8)$ & $39(50)$ & $15(19.2)$ \\
PSO view & $54(69.2)$ & $22(28.2)$ & $2(2.6)$ \\
\hline
\end{tabular}

The results are expressed in number (\%)

Table II. The frequency of facet joint degeneration in ultrasound transverse median TM view

\begin{tabular}{llll}
\hline \multirow{2}{*}{ TM view } & \multicolumn{2}{c}{ Facet joint degeneration } & \multirow{2}{*}{ p value } \\
\cline { 2 - 3 } & Yes & No & \\
\hline Good view & $15(62.5)$ & $9(37.5)$ & 0.36 \\
Intermediate view & $24(61.5)$ & $15(38.5)$ & 0.25 \\
Poor view & $3(20)$ & $12(80)$ & 0.004 \\
\hline
\end{tabular}

The results are expressed in number (\%)

highly curved surface (in this case degenerated facet joint and ligamentum flavum with soft tissues surrounding it) an artefact named lateral shadowing [3,4] (or retraction shadowing [5]) appears. Marginal waves get refracted at the edges of the structure, causing a shadow at the margins. Sound is refracted in the same way that light is as it passes from one medium to another. Thus, the direction in which it travels changes when it passes through a 
boundary at an angle less than 90 degrees. This can lead to subtle miss placement of structures and some degradation of image quality when the angle of incidence is particularly acute.

Scattering occurs when the reflecting surface is very small compared to the sonographic wavelength (degenerated facet joint) and echoes are reflected through a wide range of angles, consequently reducing their detected intensity. Also, the beams are distorted after contact with an irregular surface thereby interfering with the beams reflected from nearby structures.

Both these phenomena result in the degradation of image quality from structures deep to the irregular bony surface of degenerated facet joint. This is more relevant to images produced in TM plane where the facet joint is in the same plane as the inter-spinous space, leading to refractive shadowing and scattering. The artefacts may cause a poor image in spite of the facets being lateral and the target of interest (anterior complex and posterior complex) being medial (supplemental figure 2, on the journal site). Poor neuraxial ultrasound views in our study was strongly associated with facet joint degeneration. This might explain the high false negative rates of TM view [2]. This might also explain the fact that the PSO view did not have any correlation with facet degeneration. In the PSO view, the ultrasound beam passes through the laminae and is less subjected to the various artefacts described (supplemental figure 3, on the journal site).

Our study did not find any relation between the nonfusion of ligamentum flavum in the midline and poor views. The most probable reason was the very low incidence of ligamentum flavum gaps detected in our study population. Presence of gaps in the midline due to failure of fusion of ligamentum flavum has been reported previously in the literature. In a cytomicrotome study on 38 cadavers by Hogan et al [6], it was shown that there was a variable incidence of ligamentum flavum gaps in the lumbar region. Lirk et al, in his study on 45 cadavers, documented the incidence of ligamentum flavum gaps in the lumbar region as follows: L1L2 - 22.2\%, L2L3 11.4\%, L3L4 -11.1\%, L4L5 - 9.3\% and L5S1 - 0\% [7]. In our study we have only 2 cases of absent ligamentum flavum and in both instances the ultrasound did not visualise the LF-dura complex. This difference may be due to the fact that cadaveric studies might have overestimated its incidence. Integrity of epidural fat, thecal sac and epidural veins cannot be preserved once the tissues are dissected as the delicate balance of pressures between them are disrupted [3]. Ligamentum flavum is usually held under tension and retracts when cut which might create gaps that might not exists otherwise [8,9]. Also, the incidence of ligamentum flavum gaps are reported to be higher in cervical and high thoracic regions compared to lumbar regions [10].

Skin - posterior longitudinal ligament distance made no difference to the ultrasound views. Similarly, anatomical factors such as para-spinal muscle thickness, subcutaneous fat, epidural fat and ligamentum flavum thickness did not have any influence on the quality of view.

Facet joint degeneration can act as a surrogate marker for a number of structural changes of the spine, almost all of which might make the administration of neuraxial block difficult in a given inter-spinous space. Those include: degenerative disc disease [11-13], narrowing of intervertebral space, increase in L1-5 lumbar lordosis, pelvic incidence, sacral slope [14], associated bucking and hypertrophy of ligamentum flavum, ligamentum flavum calcification [15] and spinal canal stenosis [16].

On the other hand, PSO view do not capture the facet joint degeneration and hence its use in predicting the ease of administration of neuraxial block is limited [2].

Table III. Comparison of demographic and MRI variables between poor and non-poor visualization groups in ultrasound transverse median (TM) view

\begin{tabular}{llll}
\hline \multirow{2}{*}{ Variables } & \multicolumn{3}{c}{ Poor view in TM orientation } \\
\cline { 2 - 4 } & No $(\mathbf{n}=\mathbf{6 3})$ & Yes $(\mathbf{n}=\mathbf{1 5})$ & 0.016 \\
\hline Age (years) & $47(43-58)$ & $54(51-68)$ & 0.860 \\
Height (cm) & $160(154-166)$ & $164(152-166)$ & 0.029 \\
Weight (Kg) & $77(69-80)$ & $80(77-89)$ & 0.011 \\
BMI & $29.1(27.6-32)$ & $32.1(29.7-33.2)$ & 0.590 \\
Subcutaneous fat (mm) & $32(19.6-39.7)$ & $35.6(18.8-39.8)$ & 0.320 \\
LF thickness (mm) & $2.6(2.1-3.1)$ & $3(2.2-3.2)$ & 0.550 \\
EFP thickness (mm) & $5.1(3-7.1)$ & $4.3(2.7-7.2)$ & 0.76 \\
Skin-PLL (mm) & $77(14.4)$ & $78.6(16.6)$ & 0.16 \\
PSM thickness (mm) & $43.7(13.2)$ & $49.1(13.1)$ & 0.55 \\
Thecal sac diameter (mm) & $13.1(2.3)$ & $12.7(2.5)$ & \\
\hline
\end{tabular}

The results are expressed as median (interquartile range) or mean (standard deviation). LF - ligamentum flavum; EFP - epidural fat pad; PLL - posterior longitudinal ligament; PSM - para-spinal muscle; $\mathrm{n}$ - number 
Our study has a number of limitations. The small cohort size may have underpowered our ability to detect correlations with the measured parameters. Calcification of interspinous structures (e.g. interspinous ligament, supraspinous ligament and ligamentum flavum) cannot be accurately identified by MRI. But the incidences of such calcifications are low, ranging between $2.4 \%$ to $6.7 \%$ [17] and with regards to assessment of facet joint degeneration, MRI is as good as computer tomography $[13,18]$. Imaging positions also differed between MRI (supine) and ultrasound (sitting with back arched) which may have limited direct comparability between findings. Intervertebral disc height and dural sac diameter changes between supine and sitting position [19,20]. We believe that the magnitude of postural changes is small and will have minimal effect on the outcome of this study. Neuraxial ultrasound scanning was performed by experts and results may not reflect those obtained by novice scanners. Also, the study was done in a non-obstetric population, the results of which may not be applicable in an obstetric setting.

In conclusion, facet joint degeneration is associated with up to $80 \%$ of poor views on TM scans of spine. PSO view is not affected by any of the measured factors. Poor visualisation of ligamentum flavum/duramater complex in TM view might be due to one of the two reasons: i) the ultrasound beam is not able to reach the target or ii) the target structure is absent or defective. Our study has shown that the former, rather than the latter is the more plausible explanation in most cases, with cause being artefactual rather than structural.

Future studies on improving the neuraxial imaging should focus on postural changes to move the facet joint away from the path of the beam e.g. flexion and lateral rotation. This method has been studied before for neuraxial ultrasound in thoracic regions [20]. In future, the role of such manoeuvres to improve the neuraxial ultrasound imaging could be explored in interspinous spaces with poor visibility.

\section{Conflict of interest: none}

\section{References}

1. Weed JT, Taenzer AH, Finkel KJ, Sites BD. Evaluation of pre-procedure ultrasound examination as a screening tool for difficult spinal anaesthesia*. Anaesthesia 2011;66:925930.

2. Chin KJ, Ramlogan R, Arzola C, Singh M, Chan V. The utility of ultrasound imaging in predicting ease of performance of spinal anesthesia in an orthopedic patient population. Reg Anesth Pain Med 2013;38:3438 .
3. Steel R, Poepping TL, Thompson RS, Macaskill C. Origins of the edge shadowing artefact in medical ultrasound imaging. Ultrasound Med Biol 2004;30:1153-1162.

4. Rubin JM, Adler RS, Fowlkes JB, Carson PL. Phase cancellation: a cause of acoustical shadowing at the edges of curved surfaces in B-mode ultrasound images. Ultrasound Med Biol 1991;17:85-95.

5. Taljanovic MS, Melville DM, Scalcione LR, Gimber LH, Lorenz EJ, Witte RS. Artifacts in musculoskeletal ultrasonography. Semin Musculoskelet Radiol 2014;18:3-11.

6. Hogan QH. Epidural anatomy examined by cryomicrotome section. Influence of age, vertebral level, and disease. Reg Anesth Pain Med 1996;21:395-406.

7. Lirk P, Moriggl B, Colvin J, et al. The incidence of lumbar ligamentumflavummidlinegaps.AnesthAnal2004;98:1178-1180.

8. Zarzur E. Anatomic studies of the human ligamentum flavum. Anesth Anal 1984;63:499-502.

9. Ramsey RH. The anatomy of the ligamenta flava. Clin Orthop Relat Res 1966;44:129-140.

10. Lirk P, Kolbitsch C, Putz G, et al. Cervical and high thoracic ligamentum flavum frequently fails to fuse in the midline. Anesthesiology 2003;99:1387-1390.

11. Dunlop RB, Adams MA, Hutton WC. Disc space narrowing and the lumbar facet joints. J Bone Joint Surg Br 1984;66:706-710.

12. Jinkins JR. Acquired degenerative changes of the intervertebral segments at and suprajacent to the lumbosacral junction. A radioanatomic analysis of the nondiscal structures of the spinal column and perispinal soft tissues. Eur J Radiol 2004;50:134-158.

13. Fujiwara A, Tamai K, Yamato M, et al. The relationship between facet joint osteoarthritis and disc degeneration of the lumbar spine: an MRI study. Eur Spine J 1999;8:396-401.

14. Sahin MS, Ergun A, Aslan A. The Relationship Between Osteoarthritis of the Lumbar Facet Joints and Lumbosacropelvic Morphology. Spine (Phila Pa 1976) 2015;40:E1058E1062.

15. Schrader PK, Grob D, Rahn BA, Cordey J, Dvorak J. Histology of the ligamentum flavum in patients with degenerative lumbar spinal stenosis. Eur Spine J 1999;8:323-328.

16. Alvarez JA, Hardy RH Jr. Lumbar spine stenosis: a common cause of back and leg pain. Am Fam Pysician 1998;57:1825-1834.

17. Hameed F, Hunter DJ, Rainville J, Li L, Suri P. Prevalence of anatomic impediments to interlaminar lumbar epidural steroid injection. Arch Phys Med Rehabil 2012;93:339-343.

18. Weishaupt D, Zanetti M, Boos N, Hodler J. MR imaging and CT in osteoarthritis of the lumbar facet joints. Skeletal Radiol 1999;28:215-219.

19. Mauch F, Jung C, Huth J, Bauer G. Changes in the lumbar spine of athletes from supine to the true-standing position in magnetic resonance imaging. Spine (Phila Pa 1976) 2010;35:1002-1007.

20. Karadimas EJ, Siddiqui M, Smith FW, Wardlaw D. Positional MRI changes in supine versus sitting postures in patients with degenerative lumbar spine. J Spinal Dosord Tech 2006;19:495-500. 